\title{
Identificação de Jogos Digitais e Lógica de Programação
}

\section{Digital Game Identification and Programming Logic}

\author{
Luciana Michele Ventura ; Luciane Guimarães Batistella Bianchinia; Bernadete Lema Mazzafera ${ }^{a}$; Lisandra Costa Pereira \\ Kirnew $^{\mathrm{a}}$; Fabiana Fernandes Zani ${ }^{\mathrm{a}}$ Nielce Meneguelo Lobo da Costa ${ }^{\mathrm{b}}$ \\ ânopar, Programa de Pós-Graduação Stricto Sensu em Metodologia para Ensino de Linguagens e suas Tecnologias. PR, Brasil. \\ ${ }^{b}$ Universidade Anhanguera de São Paulo, Programa de Pós-Graduação Strcito Sensu em Ensino de Ciência e Saúde; e Programa de Pós-Graduação \\ Stricto Sensu em Educação Matemática. SP, Brasil. \\ *E-mail: fabiianafernandess@gmail.com \\ Recebido em: 02/07/2019; Aceito em: 27/09/19
}

\begin{abstract}
Resumo
A aprendizagem da Lógica de Programação, é uma das áreas em que os alunos possuem dificuldades. A utilização de ferramentas lúdicas pode ser um recurso útil no processo de ensino e aprendizagem deste conteúdo. Este estudo tem por objetivo identificar quais recursos digitais estão sendo utilizados para o ensino da Lógica de Programação. Para responder ao objetivo proposto realizou-se um estudo bibliométrico. O estudo foi realizado nos bancos de dados de teses e dissertações da CAPES e da BDTD - desenvolvida pelo Instituto Brasileiro de Informação em Ciência e Tecnologia (IBICT). Foram utilizadas as palavras-chave: Lógica de Programação, Ensino, Jogos Digitais e Piaget. Foram encontrados 197 trabalhos no período de abril a julho de 2017, excluindo os estudos repetidos nas duas bases e sete trabalhos, por serem mais antigos que não foram encontrados para consulta pública, na internet, restaram 37 estudos que abordaram a Lógica de Programação. Dos 18 trabalhos que utilizaram em suas pesquisas ferramentas digitais disponíveis no mercado, sete utilizaram a ferramenta scratch e foram analisados neste estudo.

Palavras-chave: Lógica de Programação. Jogos Digitais. Ensino.
\end{abstract}

\begin{abstract}
Learning Programming Logic is one of the areas in which students have difficulties. The use of playful tools can be a useful resource in the process of teaching and learning this content. This study aims to identify which digital resources are being used to teach Programming Logic. To answer the proposed objective, a bibliometric study was performed. The study was conducted in the CAPES and BDTD thesis and dissertation databases - developed by the Brazilian Institute of Information Science and Technology (IBICT). The keywords used were: Programming Logic, Teaching, Digital Games and Piaget. We found 197 papers from April to July 2017, excluding repeated studies in both databases and seven papers, because they were older than not found for public consultation, on the Internet, 37 studies remained that addressed the logic of programming. Of the 18 works that used digital tools available in their research, seven used the scratch tool and were analyzed in this study.
\end{abstract}

Keywords: Programming Logic. Digital Games. Teaching.

\section{Introdução}

O ensino e a aprendizagem da Lógica de Programação são de grande importância para a sociedade atual, que busca sujeitos com pensamentos criativos, flexíveis e que saibam resolver problemas.

Conteúdos importantes para a atualidade tecnológica, embora considerados como difíceis para muitos, são aqueles relacionados à Lógica de Programação, necessários para a promoção de indivíduos com pensamentos autônomos e flexíveis, a fim de poderem aprender a programar.

A construção do conhecimento, considerado pela perspectiva piagetiana, implica num processo interativo e ativo do indivíduo, no qual vários aspectos articulam-se (PIAGET, 1975). Neste contexto do qual a aprendizagem faz parte, sentir-se desafiado por algo pode ser um propulsor da atividade do indivíduo, sobretudo quando alguns conteúdos são considerados pelos alunos como difíceis.

Aprender a programar no século XXI é tão importante quanto foi aprender a ler e escrever no século passado. Em um de seus discursos, o ex-presidente dos EUA, incentivando o ensino de Ciências da Computação para todos e a importância de programar desde cedo, citou a seguinte frase: "Não apenas compre um videogame, faça um. Não apenas faça download do último aplicativo, ajude a desenvolvê-lo. Não apenas jogue no seu celular, programe!” (OBAMA, 2013).

O desenvolvimento do pensamento computacional, desde cedo, é de extrema importância para que, no futuro, existam cidadãos capazes de desenvolver as tecnologias digitais utilizadas na sociedade moderna. Costa, Pessôa e Gomes (2017) mencionam que estudantes, quando instigados a resolver um problema, procuram estratégias que permitam utilizar a criatividade para encontrar diferentes caminhos para resolver um problema, desenvolvendo, assim, o pensamento computacional.

Ensinar e incentivar os jovens a aprender programação é essencial para atender às grandes demandas da sociedade atual por recursos tecnológicos, que abrem um amplo campo para os profissionais da área da Computação. Os programadores são profissionais necessários para atuar no desenvolvimento 
de softwares, que irão atender os diferentes segmentos da sociedade.

Uma estratégia muito útil para trabalhar conteúdos da Lógica de Programação de modo significativo são os recursos lúdicos integrados às tecnologias (VALENTE, 2016).

Ramos (2013), destaca que os jogos cognitivos permitem a estimulação e realização de exercícios que têm o potencial de modificar a organização estrutural e funcional do cérebro, proporcionando a evolução das habilidades cognitivas dos jogadores.

Portanto, este estudo tem por objetivo identificar quais recursos digitais estão sendo utilizados para o ensino da Lógica de Programação.

\section{Desenvolvimento}

\subsection{Metodologia}

Para verificar os estudos sobre o ensino da Lógica de Programação, realizou-se levantamento bibliométrico, no período de Abril a Julho/2017, no qual os registros de dados foram anotados em uma planilha do Excel, de acordo com as orientações de Hayashi e Hayashi (2011), sendo adaptada às necessidades de registro da pesquisa. Os bancos de dados de teses e dissertações da CAPES e da BDTD - desenvolvida pelo Instituto Brasileiro de Informação em Ciência e Tecnologia (IBICT) - foram utilizados com o intuito de investigar publicações de dissertações e teses sobre o ensino da Lógica de Programação, na Educação Básica e em cursos da área da Computação, seja de nível técnico ou superior. Com o intuito de verificar o uso de recursos digitais, por meio de ferramentas lúdicas no ensino da Lógica de Programação, buscou-se trabalhos relacionados com os descritores: Lógica de Programação, Ensino, Jogos Digitais e Piaget (Quadro 1).

Quadro 1 - Descritores utilizados na pesquisa realizada na Base de Dados da CAPES e BDTD

\begin{tabular}{|l|c|}
\hline \multicolumn{1}{|c|}{ Termos da busca } & $\begin{array}{c}\text { Total de } \\
\text { Trabalhos }\end{array}$ \\
\hline "lógica de programação" & 66 \\
\hline "lógica de programação" AND ensino & 51 \\
\hline "lógica de programação" AND jogos & 24 \\
\hline "lógica de programação" AND Piaget & 29 \\
\hline "lógica de programação" AND ensino AND Piaget & 22 \\
\hline "lógica de programação" AND jogos AND Piaget & 5 \\
\hline
\end{tabular}

Fonte: Dados da pesquisa.

Ao todo foram localizados 197 trabalhos, somando as duas bases de dados pesquisadas. Desse total, subdividese nas seguintes combinações de descritores: "lógica de programação", a qual resultou em 66 trabalhos; "lógica de programação" AND ensino, que trouxe 51 trabalhos; "lógica de programação" AND jogos, resultou em 24 trabalhos; lógica de programação AND Piaget, trouxe como resultado 29 trabalhos; lógica de programação AND ensino AND Piaget, resultou em 22 trabalhos e, por último, lógica de programação AND jogos AND Piaget, a qual trouxe como resultado cinco trabalhos. Os resultados das buscas são apresentados quantitativamente por meio de quadros, gráficos e nuvem de palavras, como pressupõe a pesquisa bibliométrica "[...] que precisa, necessariamente, contemplar uma etapa de análise quantitativa de dados" (SOARES; PICOLLI; CASAGRANDE, 2018, p.308). Para atingir ao objetivo proposto os dados são apresentados em etapas: $1^{\text {a }}$ ) as palavras que preponderaram nos trabalhos e os trabalhos disponíveis para consulta; $2^{\mathrm{a}}$ ) os trabalhos relacionados a lógica de programação com o uso de ferramentas digitais. $3^{\mathrm{a}}$ ) a partir dos trabalhos destacam-se e apresentam-se: o período de maior publicação; as ferramentas digitais presentes nos trabalhos; a divisão dos trabalhos por área de estudo, região do país e instituição; análise dos trabalhos que utilizaram o Scratch (ferramenta que preponderou). $4^{\mathrm{a}}$ ) os trabalhos que utilizaram a ferramenta Scratch são apresentados e analisados.

\subsection{As palavras que preponderaram nos trabalhos localizados}

O Nuvem de Palavras, a seguir, relaciona as palavraschave que aparecem nos trabalhos localizados na pesquisa realizada nas bases de dados da CAPES e BDTD.

As palavras-chave que definiram esses trabalhos podem ser divididas em três grupos: Ensino e Educação, Uso de Tecnologia na Educação e Ensino da Lógica de Programação.

As palavras agrupadas em Ensino e Educação foram: Aprendizagem Baseada em Casos, Aprendizagem Significativa, Aprendizagem, Autoavaliação, Autorregulação da Aprendizagem, Avaliação por Pares, Ciência, Construção do Conhecimento, Construcionismo, Construtivismo, Educação Básica, Educação, Ensino, Ensino auxiliado por Computador, Ensino de Matemática, Ensino Fundamental, Ensino-Aprendizagem, Estudo e Ensino, Física, Inovações Educacionais, Interdisciplinaridade, Jogos Didáticos, Lúdico, Matemática, Metacognição, Metodologia em Educação, Motivação, Prática de Ensino, Prática Pedagógica, Projeto de Trabalho, Raciocínio Baseado em Casos, Resolução de Problemas e Semiótica Organizacional. (Figura 1).

Figura 1 - Nuvem de Palavras - Palavras-chaves encontradas nos trabalhos

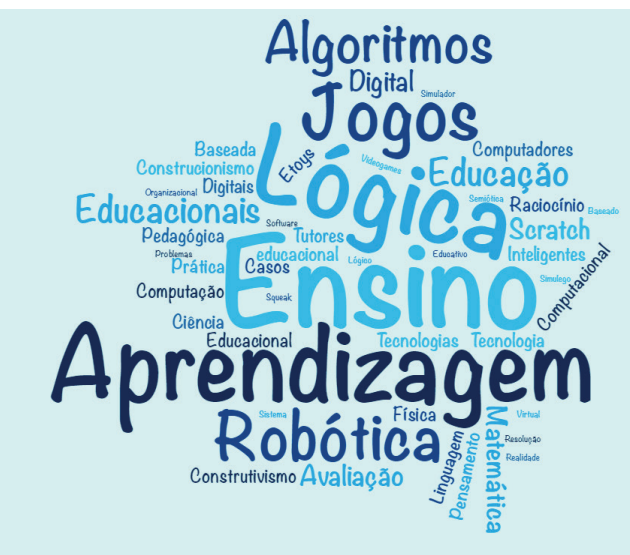

Fonte: Os autores. 
Neste item, pode-se perceber que as palavras relacionamse fundamentalmente com a aprendizagem. Além disso, tais palavras inferem sobre alguns aspectos como motivação, conteúdos (física, matemática), metodologias de ensino e atividade lúdica.

Outro agrupamento possível refere-se ao uso de Tecnologia na Educação: Ambientes Educacionais Informatizados, Cultura Digital, Ferramenta, Gamificação, Hibridismo Tecnológico, Informática na Educação, Inteligência Artificial, Jogos Digitais, Jogos Educacionais, Jogos Educativos, Jogos Eletrônicos, Jogos Sérios, Literacia Digital, Mineração de Dados Educacionais, Sistema Tutores Inteligentes, Squeak Etoys, Tecnologia Educacional, Tecnologias, Tecnologias Digitais, Videogames, Fluência Digital e FUNSoftWare RCX2.

Por fim, as palavras-chave podem ser agrupadas em Ensino da Lógica de Programação: Algoritmos, Lógica de Programação, Ensino de Linguagem de Programação, Pensamento Computacional, Ciência da Computação, Framework, Filosofia LOGO, Scratch, App Inventor, Blockly, Laboratório Remoto de Robótica, LEGO, Robótica, Robótica Educacional, Robótica Pedagógica, Simulador e Simulego.

A pesquisa teve um resultado inicial com os descritores citados, nas duas bases de dados (CAPES e BDTD), totalizando 197 trabalhos (Quadro 2).

Quadro 2 - Total de Trabalhos Encontrados

\begin{tabular}{|l|c|}
\hline Trabalhos & Quantidade \\
\hline Total de trabalhos encontrados & 197 \\
\hline Trabalhos repetidos & 97 \\
\hline Trabalhos fora da área pesquisada & 56 \\
\hline Trabalhos indisponíveis para consulta pública & 07 \\
\hline Total de trabalhos para tabulação & $\mathbf{3 7}$ \\
\hline
\end{tabular}

Fonte: Dados da pesquisa.

Como se pode verificar no Quadro 2, 97 trabalhos repetidos e 56 fora da área de interesse dessa pesquisa, ou seja, não se tratavam de estudos sobre o ensino da Lógica de Programação, sete dos trabalhos, por serem mais antigos não foram encontrados para consulta pública, por meio da internet, restando 37 trabalhos para este estudo.

\subsection{Trabalhos relacionados com o ensino da Lógica de Programação, por meio de ferramentas digitais}

Para atender aos requisitos da pesquisa e objetivo deste estudo, foram encontrados 37 trabalhos relacionados ao ensino da Lógica de Programação, por meio de ferramentas digitais, apenas um desses trabalhos não utilizou recurso digital para a pesquisa.

Quanto ao nível da pesquisa, foram localizadas quatro teses e 33 dissertações, as quais tiveram a última década como o período com maior desenvolvimento de trabalhos relacionados ao tema, 25 dos trabalhos foram realizados de 2010 a 2017 (Gráfico 1).
Gráfico 1 - Divisão dos trabalhos publicados por período

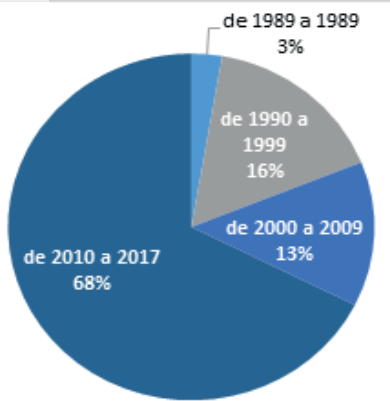

[ de 1989 a 1989

a de 1990 a 1999

- de 2000 a 2009

a de 2010 a 2017

Fonte: Dados da pesquisa.

A análise dos trabalhos encontrados trouxe um dado interessante: 22 pesquisas fizeram uma intervenção com aplicação prática utilizando jogos ou ferramentas digitais para ensinar a Lógica de Programação. Entre essas 22 intervenções, apenas uma não utilizou recurso digital. Percebe-se um grande interesse nas pesquisas em verificar a aplicabilidade de ferramentas digitais e a ludicidade na introdução da Lógica, tanto para alunos do Ensino Fundamental, como do Ensino Médio, Ensino Técnico ou Ensino Superior.

\subsubsection{Ferramentas digitais presentes nos trabalhos}

$\mathrm{Na}$ pesquisa realizada, verificou-se a existência de trabalhos envolvendo o desenvolvimento de Ambiente Gamificado, Framework, Jogo e Software. Assim como o uso de ferramentas digitais disponíveis no mercado: Alice, App Inventor, Blocky, Etoys, Lego, Linguagem Logo, Raptor, Scratch, Visual G, a Robótica e o website learn.code.org.

Quadro 3 - Total de trabalhos encontrados, separados por recurso digital utilizado

\begin{tabular}{|l|c|}
\hline \multicolumn{1}{|c|}{ Recurso Digital } & $\begin{array}{c}\text { Quantidade de } \\
\text { Trabalhos }\end{array}$ \\
\hline Blocky e App Inventor & 1 \\
\hline Desenvolvimento de Ambiente Gamificado & 1 \\
\hline Desenvolvimento de Framework & 1 \\
\hline Desenvolvimento de Jogo & 2 \\
\hline Desenvolvimento de Software & 13 \\
\hline Etoys & 1 \\
\hline Linguagem Logo & 4 \\
\hline Linguagem Scratch & 4 \\
\hline Linguagem Scratch e Alice & 1 \\
\hline Linguagem Scratch, Visual G e Lego & 1 \\
\hline Linguagem Scratch e Raptor & 1 \\
\hline Robótica & 5 \\
\hline Website learn.code.org & 1 \\
\hline
\end{tabular}

Fonte: Dados da pesquisa.

Ao analisar o material de pesquisa identificou-se (Quadro 3), o uso de 10 diferentes ferramentas digitais nos 37 trabalhos, distribuídas em jogos, linguagens de programação e website, destinados ao ensino da Lógica de Programação. Alguns trabalhos relatam o uso de mais de uma ferramenta.

Também encontrou-se trabalhos que relatam o desenvolvimento de softwares específicos para a aplicação daquela pesquisa, num total de 18 nessa situação. 
Observou-se que, entre os recursos digitais, destacou-se a linguagem Scratch, a qual apareceu em sete dos 18 trabalhos que utilizaram em suas pesquisas ferramentas digitais disponíveis no mercado.

Quadro 4 - Linguagens, Softwares e Websites disponíveis no mercado, que foram utilizados nos trabalhos encontrados

\begin{tabular}{|l|c|}
\hline \multicolumn{1}{|c|}{ Linguagens, Softwares e Websites } & Quantidade \\
\hline Alice & 1 \\
\hline App Inventor & 1 \\
\hline Blocky & 1 \\
\hline Etoys & 1 \\
\hline Linguagem Logo & 4 \\
\hline Linguagem Scratch & 7 \\
\hline Raptor & 1 \\
\hline Robótica & 5 \\
\hline Visual G & 1 \\
\hline Website learn.code.org & 1 \\
\hline
\end{tabular}

Fonte: Dados da pesquisa.

Pode-se observar (Quadro 4), que entre os 18 trabalhos que utilizaram linguagens, softwares e websites disponíveis no mercado e de fácil acesso por meio da internet, 7 deles utilizaram como recurso digital para o ensino da Lógica de Programação a linguagem Scratch.

2.3.2 Divisão dos trabalhos por área de estudo, região do país e instituição

Os trabalhos que atenderam aos requisitos desta pesquisa podem ser agrupados em seis áreas de estudo: Design, Educação e Ensino, Engenharia, Informática e Computação, Informática na Educação e Psicologia (Gráfico 2).

Gráfico 2 - Trabalhos agrupados por área de estudo

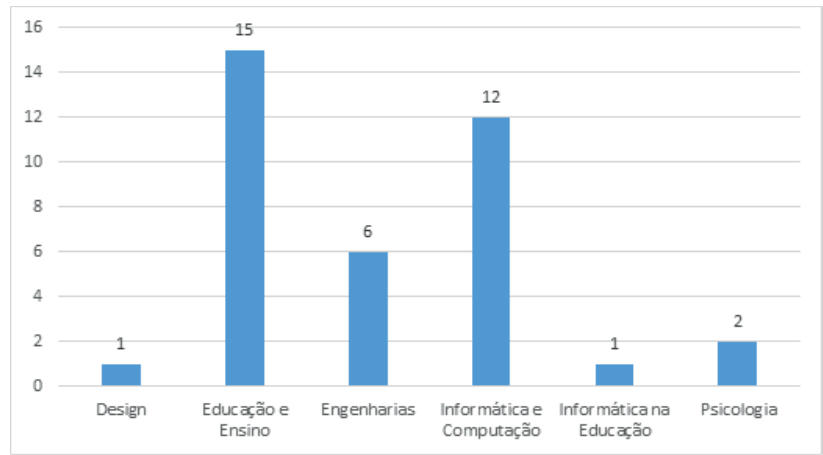

Fonte: Dados da pesquisa.

Essas áreas de estudo fazem parte dos Programas de Pós-Graduação Stricto Sensu de instituições do país que se apresentam divididos em quatro dependências administrativas (Quadro 5), sendo 1 Comunitária, 3 Estaduais, 19 Federais, 13 Privadas e 1 Programa Internacional, da Universidade de Coimbra.
Quadro 5 - Instituições pesquisadoras divididas em dependências administrativas

\begin{tabular}{|l|c|}
\hline Instituições Pesquisadoras & Quantidade \\
\hline Comunitária & 1 \\
\hline Estadual & 3 \\
\hline Federal & 19 \\
\hline Privada & 13 \\
\hline Internacional & 1 \\
\hline Total & $\mathbf{3 7}$ \\
\hline
\end{tabular}

Fonte: Dados da pesquisa.

A pesquisa também apontou que a região do país (Gráfico 3) onde está sendo realizada a maior parte dos estudos sobre Lógica de Programação, utilizando recursos digitais, é a região Sul, com 17 trabalhos; em seguida está a região Sudeste, com 11 trabalhos; a região Nordeste produziu sete trabalhos; e na região Norte, apenas um trabalho sobre o tema; e um trabalho Internacional.

Gráfico 3 - Divisão dos trabalhos por regiões do Brasil

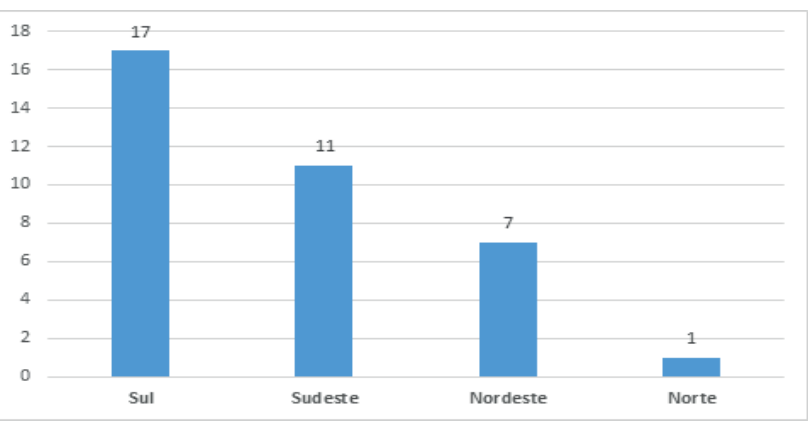

Fonte: Dados da pesquisa.

O Gráfico 3, mostra a concentração dos trabalhos localizados nas regiões Sul e Sudeste do país, as quais somam um total de $80 \%$ dos estudos. Com base nos estudos encontrados identificou-se o jogo/ferramenta lúdica Scratch, que será apresentado no item seguinte.

\subsubsection{Análise dos trabalhos que utilizaram o Scratch}

Dos sete trabalhos encontrados que utilizaram a linguagem Scratch, cinco vieram das combinações de descritores "lógica de programação" e dois vieram da combinação "lógica de programação AND Piaget", todos os trabalhos foram encontrados na base de dados da Capes. O Quadro 6, traz uma síntese dos dados dessas sete pesquisas. 
Quadro 6 - Relação dos trabalhos encontrados que utilizaram a linguagem Scratch

\begin{tabular}{|c|c|c|c|c|}
\hline Título do Trabalho & $\begin{array}{c}\text { Autor/ } \\
\text { Pesquisador }\end{array}$ & $\begin{array}{l}\text { Local da } \\
\text { Pesquisa }\end{array}$ & $\begin{array}{c}\text { Área de } \\
\text { Conhecimento }\end{array}$ & $\begin{array}{l}\text { Ferramenta } \\
\text { Utilizada }\end{array}$ \\
\hline $\begin{array}{l}\text { Uma investigação do uso da ferramenta Scratch } \\
\text { para o ensino de Lógica de Programação no } \\
\text { Ensino Médio }\end{array}$ & $\begin{array}{c}\text { Renanh } \\
\text { Gonçalves de } \\
\text { Araújo } \\
\end{array}$ & $\begin{array}{l}\text { Fortaleza } \\
\quad \text { CE }\end{array}$ & Informática & Scratch \\
\hline $\begin{array}{l}\text { Ensino de Algoritmo e Lógica de Programação: } \\
\text { Modelo Construtivista auxiliado pelo Scratch }\end{array}$ & $\begin{array}{l}\text { Flamarion Assis } \\
\text { Jeronimo Inácio }\end{array}$ & $\begin{array}{l}\text { Uberaba } \\
\text { MG }\end{array}$ & Educação & Scratch \\
\hline $\begin{array}{l}\text { O uso da Lógica de Programação para a Educação } \\
\text { Matemática no Ensino Médio: experiências com o } \\
\text { Scratch }\end{array}$ & $\begin{array}{c}\text { Samantha Pinto } \\
\text { da Silva }\end{array}$ & $\begin{array}{l}\text { Pelotas } \\
\text { RS }\end{array}$ & Ensino & Scratch \\
\hline $\begin{array}{l}\text { A construção do conhecimento de Algoritmos no } \\
\text { contexto do Hibridismo Tecnológico: Análise da } \\
\text { prática pedagógica aplicada no IFRS }\end{array}$ & $\begin{array}{c}\text { Fabricia Py } \\
\text { Tortelli Noronha }\end{array}$ & $\begin{array}{c}\text { Canoas } \\
\text { RS }\end{array}$ & Educação & $\begin{array}{l}\text { Lego, Scratch e } \\
\text { VisualG. }\end{array}$ \\
\hline $\begin{array}{l}\text { As potencialidades do uso do software Scratch } \\
\text { para a construção da literacia digital }\end{array}$ & $\begin{array}{l}\text { Ramon dos Santos } \\
\text { Lummertz }\end{array}$ & $\begin{array}{l}\text { Canoas } \\
\text { RS }\end{array}$ & Ensino & Scratch \\
\hline $\begin{array}{l}\text { Usando o Scratch para potencializar o pensamento } \\
\text { criativo em crianças do ensino fundamental }\end{array}$ & $\begin{array}{l}\text { Amilton Rodrigo } \\
\text { de Quadros } \\
\text { Martins }\end{array}$ & $\begin{array}{c}\text { Passo Fundo } \\
\text { RS }\end{array}$ & Educação & Scratch \\
\hline $\begin{array}{l}\text { Sistematização da aprendizagem de programação } \\
\text { em grupo }\end{array}$ & $\begin{array}{c}\text { Thaís Helena } \\
\text { Chaves de Castro }\end{array}$ & $\begin{array}{l}\text { Rio de Janeiro } \\
\text { RJ }\end{array}$ & Informática & Raptor e Scratch \\
\hline
\end{tabular}

Fonte: Dados da pesquisa.

Vale destacar que, dos trabalhos indicados no Quadro 6, quatro vieram da região Sul do Brasil. As sete pesquisas indicadas correspondem ao período de 2011 a 2016, e cinco delas foram realizadas no ano de 2016. A maior parte das pesquisas foram realizadas em projetos desenvolvidos em instituições privadas do país, num total de cinco dos sete trabalhos descritos.

O resultado da busca na qual a ferramenta Scratch aparece em sete trabalhos, em todos eles foram realizadas aplicações práticas com alunos do Ensino Fundamental, Ensino Médio, Ensino Técnico Integrado ao Médio e no Ensino Superior (Quadro 7).

Quadro 7 - Aplicação dos trabalhos por segmentos do ensino

\begin{tabular}{|l|c|}
\hline \multicolumn{1}{|c|}{ Segmentos do Ensino } & Quantidade \\
\hline Ensino Fundamental & 2 \\
\hline Ensino Médio & 1 \\
\hline Ensino Médio Integrado ao Técnico & 1 \\
\hline Ensino Superior & 3 \\
\hline
\end{tabular}

Fonte: Dados da pesquisa.

Na sequência, elaborou-se um breve resumo de cada um dos sete trabalhos, a fim de demonstrar como utilizaram a linguagem Scratch, como ferramenta de apoio ao ensino.

O trabalho intitulado Uma Investigação do Uso da Ferramenta Scratch para o Ensino de Lógica de Programação no Ensino Médio, de Araújo (2016), traz uma experiência realizada no ensino da Lógica de Programação, em um curso de Ensino Médio Integrado ao Técnico em Informática mesmo segmento de ensino investigado na presente pesquisa -, com o principal objetivo de investigar o impacto do uso da ferramenta Scracht na aprendizagem da disciplina de Lógica de Programação.

A pesquisa intitulada Ensino de algoritmo e lógica de programação: modelo construtivista auxiliado pelo 'scratch', realizada por Inácio (2016), descreve um estudo em uma instituição de Ensino Superior, no curso de Análise e Desenvolvimento de Sistemas, na disciplina Algoritmo e Lógica de Programação (ALP), cujo principal objetivo foi:

[...] realizar um estudo dos aspectos positivos e negativos das principais metodologias utilizadas no ensino de ALP, fazendo um comparativo com as metodologias de ensino utilizadas por uma instituição de ensino superior e pública, durante a realização da disciplina de ALP das turmas de Análise de Desenvolvimento de Sistemas de 2010 até 2014. Após a análise, aplicar e coletar os resultados da utilização de uma ferramenta computacional [...] o Scratch, na disciplina de ALP, da instituição, visando melhorar o aprendizado na disciplina. (INÁCIO, 2016, p. 18).

Silva (2016) pesquisou O Uso da Lógica de Programação para a Educação Matemática no Ensino Médio: experiências com o Scratch. O trabalho foi realizado com uma turma da $3^{\text {a }}$ série do Ensino Médio de uma escola pública, com o intuito de investigar como o ensino da Lógica de Programação, por meio da linguagem Scratch, pode potencializar o ensino da Matemática para à resolução de problemas. O estudo foi alicerçado em uma perspectiva socioconstrutivista, para propiciar a mediação e colaboração, tendo em vista que o estudante da atualidade é um nativo digital.

Noronha (2016) identificou como desafio a promoção de novas práticas pedagógicas para diminuir a evasão de alunos, assim como a reprovação na disciplina de Lógica de Programação. Com o estudo intitulado A construção do conhecimento de algoritmos no contexto do hibridismo tecnológico: análise da prática pedagógica aplicada no IFRS, objetivou verificar "as potencialidades e os limites da utilização das tecnologias analógicas (caneta e papel) e digitais (VisuAlg, Scratch e kit Lego) para a construção do conhecimento de algoritmos" (NORONHA, 2016, p.13).

Lummertz (2016), em sua pesquisa As potencialidades do uso do software Scratch para a construção da literacia digital, promoveu a aplicação do Scratch com alunos do $4^{\circ}$ 
ano do Ensino Fundamental, com o objetivo de "investigar as potencialidades do uso do software de programação Scratch na constituição de aspectos relacionados à Literacia Digital e ao pensamento computacional por meio da construção de jogos eletrônicos" (LUMMERTZ, 2016, p.18). Os resultados indicaram três pontos importantes: possibilidades quanto à Literacia Digital, potencialidades sobre a elaboração do Pensamento Computacional e capacidade de associar conteúdos da Matemática indicados nos Parâmetros Curriculares Nacionais (PCN).

Martins (2012) também pesquisou o Scratch no trabalho que denominou Usando o 'Scratch' para potencializar o pensamento criativo em crianças do ensino fundamental. A pesquisa envolveu seis estudantes, divididos em três duplas, alunos do $6^{\circ}$ ano do Ensino Fundamental de uma escola municipal, utilizando a ferramenta Scratch com o objetivo de “investigar como se dá o comportamento de jovens estudantes diante do uso do computador e ambientes de programação, observando o despertar da criatividade a sua potencialidade" (MARTINS, 2012, p.7).

Com o intuito de auxiliar o processo de aprendizagem da programação em grupo, Castro (2011) realizou um estudo de caso em uma Universidade Federal denominado Sistematização da Aprendizagem de Programação em Grupo. Neste estudo, investigou plataformas de aprendizagem (moodle) utilizadas pelos alunos em períodos anteriores à pesquisa com o objetivo de analisar as interações entre os grupos on-line criados por diferentes turmas. Entre as ferramentas utilizadas pelos alunos, o autor identificou o Scratch como uma das ferramentas que possibilitou o estudo introdutório da Lógica de Programação, por meio de uma linguagem estruturada.

Como foi possível notar, os estudos destacados acima mostram diferentes pesquisas sobre o ensino da Lógica de Programação, os quais utilizaram o Scratch como ferramenta de aprendizagem diferenciada, buscando novas formas de ensino da Lógica.

De modo geral, os estudos de Lummertz (2016) e Silva (2016) destacaram que o Scratch pode ser uma ferramenta útil para o ensino da matemática, assim como da Lógica de Programação. A linguagem Scratch foi enfatizada nos estudos de Araújo (2016) e Inácio (2016), em seus aspectos afetivos motivacionais, isto porque essa ferramenta desperta o interesse do aluno convocando a ações ativas e interativas durante o ensino da Lógica de Programação. Em relação aos aspectos cognitivos, Martins (2012), concluiu que o uso do Scratch possibilita o estímulo do pensamento criativo.

Noronha (2016) concluiu que a ferramenta Scratch, por suas possibilidades variadas de aplicação ao aluno, pode enriquecer a prática do professor em seus aspectos pedagógicos para o ensino da Lógica de Programação.

\section{Conclusão}

Neste estudo foram encontrados 197 trabalhos nas bases de dados de teses e dissertações: a) 37 estudos abordaram a Lógica de Programação, quatro teses e 33 dissertações;

b) A maior parte dos estudos foi produzida em instituições federais (19) ou privadas (13);

c) Houve predomínio de estudos desenvolvidos nas regiões sul e sudeste do país onde se encontram estas instituições;

d) 18 estudos utilizaram ferramentas digitais disponíveis no mercado em suas análises e sete utilizaram a ferramenta scratch e foram analisados neste estudo.

Sobre os estudos analisados verificou-se que dois foram desenvolvidos em programas stricto-sensu de informática, dois em programas de ensino e três em programas de educação. Os estudos consideraram que a ferramenta Scratch é útil porque enriquece a prática do professor e desperta o interesse do aluno pelo conteúdo a ser ministrado.

A atividade lúdica é fator motivacional para os alunos, porém não pode prescindir do professor mediador no processo.

\section{Referências}

ARAÚJO, R.G. Uma investigação do uso da ferramenta SCRATCH para o ensino de lógica de programação no Ensino Médio. 2016147 f. Dissertação (Mestrado em Informática Aplicada) - Universidade de Fortaleza, Fortaleza, 2016.

CASTRO, T.C. Sistematização da aprendizagem de programação em grupo. 2011. 154 f. Tese (Doutorado em Informática) Pontifícia Universidade Católica do Rio de Janeiro, Rio de Janeiro, 2011.

COSTA, S.S.F.; PESSÔA, M.T.R.; GOMES, A.J. Pensamento Computacional e educação: uma experiência com Scratch no primeiro ciclo do ensino básico. In: YAEGASHI, S.F.R. et al. (Org.). Novas tecnologias digitais: reflexões, sobre mediação, aprendizagem e desenvolvimento. Curitiba: Curitiba, 2017. p.271-286.

HAYASHI, M.C.P.I.; HAYASHI, C.RM. Protocolo para coleta de dados bibliométricos em bases de dados. 2011. Mimeografado.

INÁCIO, F.A.J. Ensino de Algoritmos e Lógica de Programação: modelo Construtivista auxiliado pelo Scratch. 2016. $114 \mathrm{f}$. Dissertação (Mestrado Profissional em Educação Tecnológica) Instituto Federal de Educação, Ciência e Tecnologia do Triângulo Mineiro, Uberaba, 2016.

LUMMERTZ, R.S. As potencialidades do uso do software para a construção da literacia digital. 2016. 131 f. Dissertação (Mestrado em Ensino de Ciências e Matemática) - Universidade Luterana do Brasil, Canoas, 2016.

MARTINS, A.R.Q. Usando o Scratch para potencializar o pensamento criativo em crianças do ensino fundamental. 2012. 113 f. Dissertação (Mestrado em Educação) - Universidade de Passo Fundo, Passo Fundo, 2012.

NORONHA, F.P.T. A construção do conhecimento de algoritmos no contexto do hibridismo tecnológico: análise da prática pedagógica aplicada no IFRS. 2016. 133 f. Dissertação (Mestrado em Educação) - Centro Universitário La Salle, Canoas, 2016.

OBAMA, B. Don't just play on your phone, program it. The White House Blog. 2013. Disponível em: http://m.whitehouse. gov/blog/2013/12/09/don-t-just-play-your-phoneprogram-it. Acesso em: 27 jun. 2019.

PIAGET, J. A formação do símbolo na criança: imitação, jogo e sonho, imagem e representação. Rio de Janeiro: Zahar, 1975.

RAMOS, D. K. Jogos cognitivos eletrônicos: contribuições à aprendizagem no contexto escolar. Ciênc. Cognição, v. 18, n. 1, 
p. $19-32,2013$.

SOARES, S.V.; PICOLLI, I.R.A.; CASAGRANDE, J.L. Pesquisa bibliográfica, pesquisa bibliométrica, artigo de revisão e ensaio teórico em administração e contabilidade. Adm. Ensino Pesq., v.19, n.2 p.308-339, 2018

SILVA, S. P. O uso da lógica de programação para a Educação Matemática no Ensino Médio: experiências com o Scratch.
2016. 133 f. Dissertação (Mestrado em Ensino de Matemática) - Faculdade de Educação, Universidade Federal de Pelotas, Pelotas, 2016.

VALENTE, J.A. Integração do pensamento computacional no currículo da Educação Básica: diferentes estratégias usadas e questões de formação de professores e avaliação do aluno. e-Curriculum, v.14, n.3, p.864-897, 2016. 